\title{
About possibility to locate an EQ epicenter using parameters of ELF/ULF preseismic emission
}

\author{
A. Y. Schekotov ${ }^{1}$, O. A. Molchanov ${ }^{1}$, M. Hayakawa ${ }^{2}$, E. N. Fedorov ${ }^{1}$, V. N. Chebrov ${ }^{3}$, V. I. Sinitsin ${ }^{3}$, E. E. Gordeev ${ }^{4}$, \\ S. E. Andreevsky ${ }^{1}$, G. G. Belyaev ${ }^{1}$, N. V. Yagova $^{1}$, V. A. Gladishev ${ }^{1}$, and L. N. Baransky ${ }^{1}$ \\ ${ }^{1}$ Institute of Physics of the Earth Russian Academy of Sciences, 10, Bolshaya Gruzinskaya, 123995 Moscow D-242, Russia \\ ${ }^{2}$ Department of Electronics Engineering, The University of Electro-Communication, 1-5-1, Chofugaoka, Chofu-shi, \\ Tokyo, 182, Japan \\ ${ }^{3}$ Institute of Geophysical Survey, Russian Academy of Sciences, Far-East Branch, Petropavlovsk-Kamchatski, \\ Kamchatka, Russia \\ ${ }^{4}$ Institute of Volcanology, Petropavlovsk-Kamchatski, Kamchatka, Russia
}

Received: 14 July 2008 - Revised: 24 September 2008 - Accepted: 24 September 2008 - Published: 24 November 2008

\begin{abstract}
A relation between parameters of preseismic ULF/ELF emissions and EQ is studied. The magnetic data measured at Karymshino station (Kamchatka, Russia) along with data on local seismic activity during eight years of observations (2001-2008) are taken for the analysis. Source azimuth is detected in different techniques, based on the analysis of the total field and its polarized pulsed component. The latter technique shows a better accuracy in the source azimuth detection. The errors of the method are associated with existence of non-seismic sources and with use of onepoint observation. The second error can be eliminated by development of multi-point observations.
\end{abstract}

\section{Introduction}

There are quite many short-term earthquake (EQ) precursors (duration of several days or several weeks), which are more or less locally distributed around future epicenter (e.g., Molchanov and Hayakawa, 2008). Hence, comparative analysis of preseismic behavior of any physical field can be used to localize a region of future EQ. An accuracy of source detection is controlled by the spatial distribution of preseismic activity, and density of observations. The accuracy can be improved by taking into account any physical information about the precursory spatial distribution. For example,

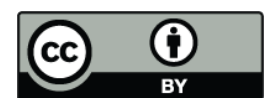

Correspondence to: A. Y. Schekotov (checkit@post.ru)
Alperovich et al. (2006) used for this purpose the difference in spatial coherence of magnetospheric and seismic ULF disturbances. The other technique is based on the direct detection of ULF seismogenic signal (Kopytenko et al., 2006). The problem becomes more sophisticated if the signal source is in the atmosphere (Oike and Yamada, 1994; Fujinawa et al., 1999). In this paper we study a possibility to detect an EQ epicenter using the preseismic emissions in the ULF/ELF frequency range. More than seven years of data collected at Karymshino station $\left(52.83^{\circ} \mathrm{N}, 158.13^{\circ} \mathrm{E}\right.$, Kamchatka, Russia) are used for the analysis. The details of observation and method of EQ time estimation are given by Schekotov et al. (2007). The plan of the paper is as follows: next section gives the description of observational facilities and data processing, section three gives the results for individual cases and for the whole period of observations. The results are discussed in the last section.

\section{Measurements and data processing}

\subsection{Observational facilities}

A detailed description of ULF magnetometer in Karymshino is given in Schekotov et al. (2007). The observatory is located at Lat $=52.827^{\circ} \mathrm{N}$, Long $=158.132^{\circ} \mathrm{E}$, continuous measurements are available since June, 2000. It is equipped with the three-component induction magnetometer in the frequency band $0.003-40 \mathrm{~Hz}$, noise level $0.16 F^{-1} \mathrm{pT} / \mathrm{Hz}^{-1 / 2}$ and conversion function $0.4 F \mathrm{~V} / \mathrm{nT}$ in the frequency band

Published by Copernicus Publications on behalf of the European Geosciences Union. 


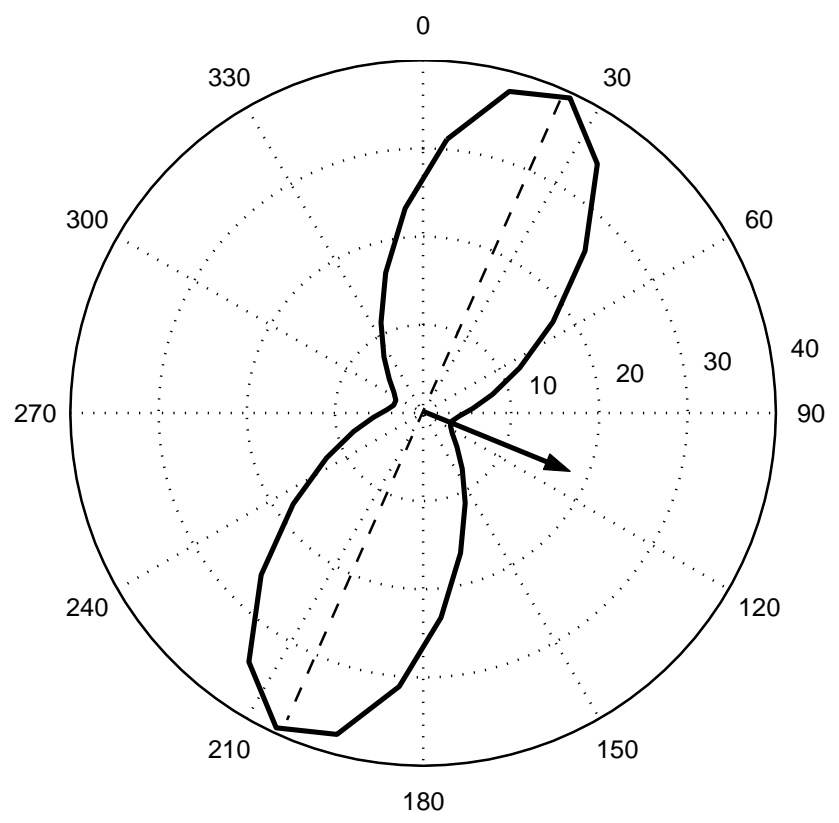

Fig. 1. An example of the distribution of $\Delta S(\varphi)$, averaged over $24 \mathrm{~h}$, in the frequency band $4-6 \mathrm{~Hz}$

$F=0.003-4 \mathrm{~Hz}$ and $1.6 \mathrm{~V} / \mathrm{nT}$ in the band $F=4-40 \mathrm{~Hz}$. The sensors for the horizontal components $H$ and $D$ are oriented along the magnetic meridian and transversally to it, and the $\mathrm{Z}$ sensor is vertical.

\subsection{Data processing}

The EQs with $\mathrm{Ms} \geq 5.7$ and distance from the observation point $\leq 500 \mathrm{~km}$ are taken for the analysis. There are 18 events with the proper quality of magnetic data during the period of observations. As it has been shown Schekotov et al. (2007) the frequency range $4-6 \mathrm{~Hz}$ is optimal for the analysis of preseismic ELF emissions.

Also as in (Schekotov et al., 2007), we introduce a parameter determinancy $\Delta S$, which is defined via spectral and polarization parameters as

$\Delta S=\frac{P_{h h} / P_{d d}-1}{\operatorname{rms}(\tan \beta)}$

where $P_{h h}, P_{d d}, P_{h d}$, and $P_{d h}$ are the elements of the polarization matrix, and

$\beta=\frac{1}{2} \arcsin \left\{\frac{\operatorname{Im}\left(P_{d h}-P_{h d}\right)}{\left[\left(P_{h h}-P_{d d}\right)^{2}+4 P_{h h} P_{d d}\right]^{1 / 2}}\right\}$.

The numerator in the righthand of Eq. (1) contains the ratio of spectral components $P_{h h}(\mathrm{~N}-\mathrm{S})$ and $P_{d d}(\mathrm{E}-\mathrm{W})$. A more general approach should be based on the coordinate system,
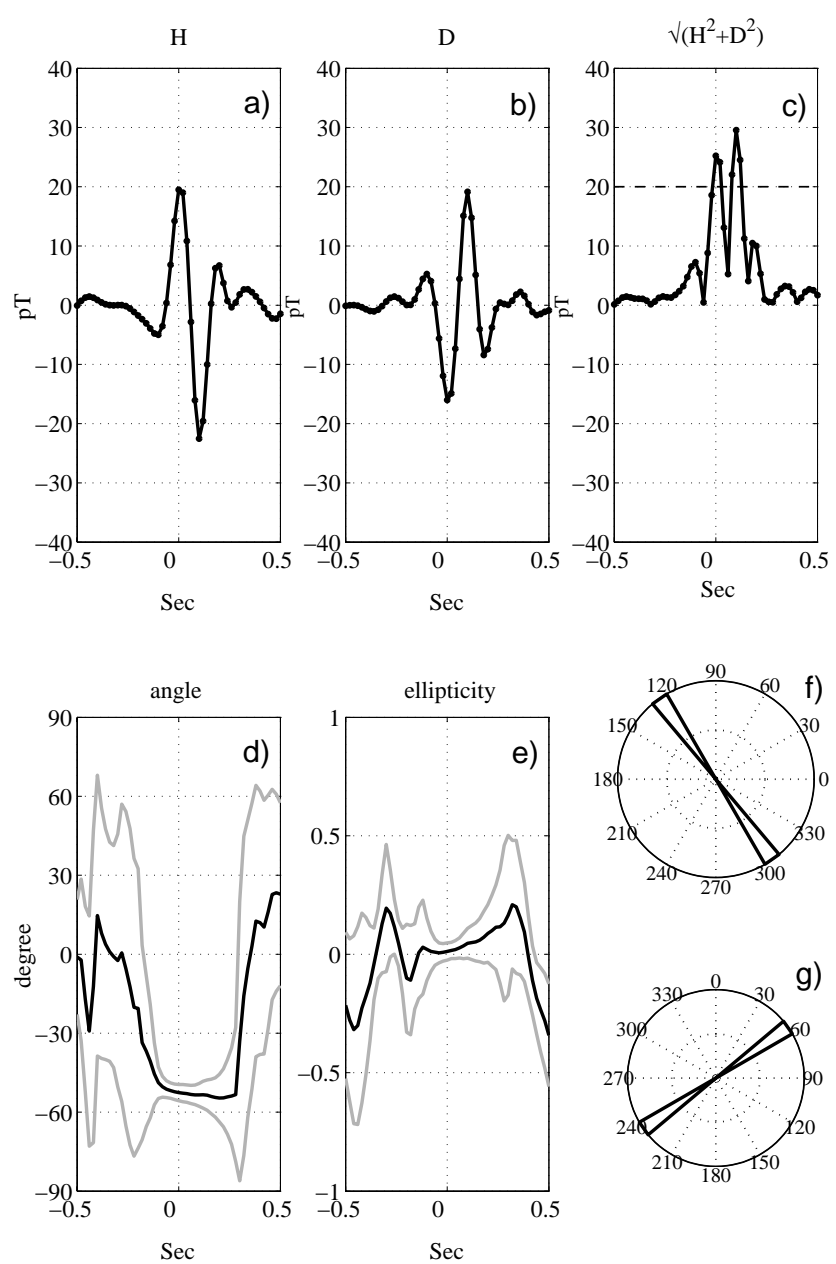

Fig. 2. An example of data processing. Panels (a) and (b) show records for $\mathrm{H}$ and $\mathrm{D}$ components, respectively, and panel (c) shows effective total amplitude and amplitude threshold (dashed horizontal line). Panels (d) and (e) show the big axis angle and ellipticity in time domain (gray lines give root mean square deviation). Panels (f) and (g) show the big axis angle and the source azimuth distributions averaged over intervals with amplitudes exceeding threshold.

connected with the source azimuth. Actually, Eq. (1) was successfully used in (Schekotov et al., 2007), because the majority of earthquakes take place eastward from the observational point. A more general approach is developed in the next section. The ellipticity, or the ratio of minor to major axis, is defined by $|\tan \beta|$. If $\beta>0, \beta<0$, or $\beta=0$ the polarization is right-hand (RH), left-handed (LH), or linear, respectively. The denominator in Eq. (1) is the root mean square deviation of the signal ellipticity. This parameter proved to be the most sensitive and reproducible to the seismic shocks. 

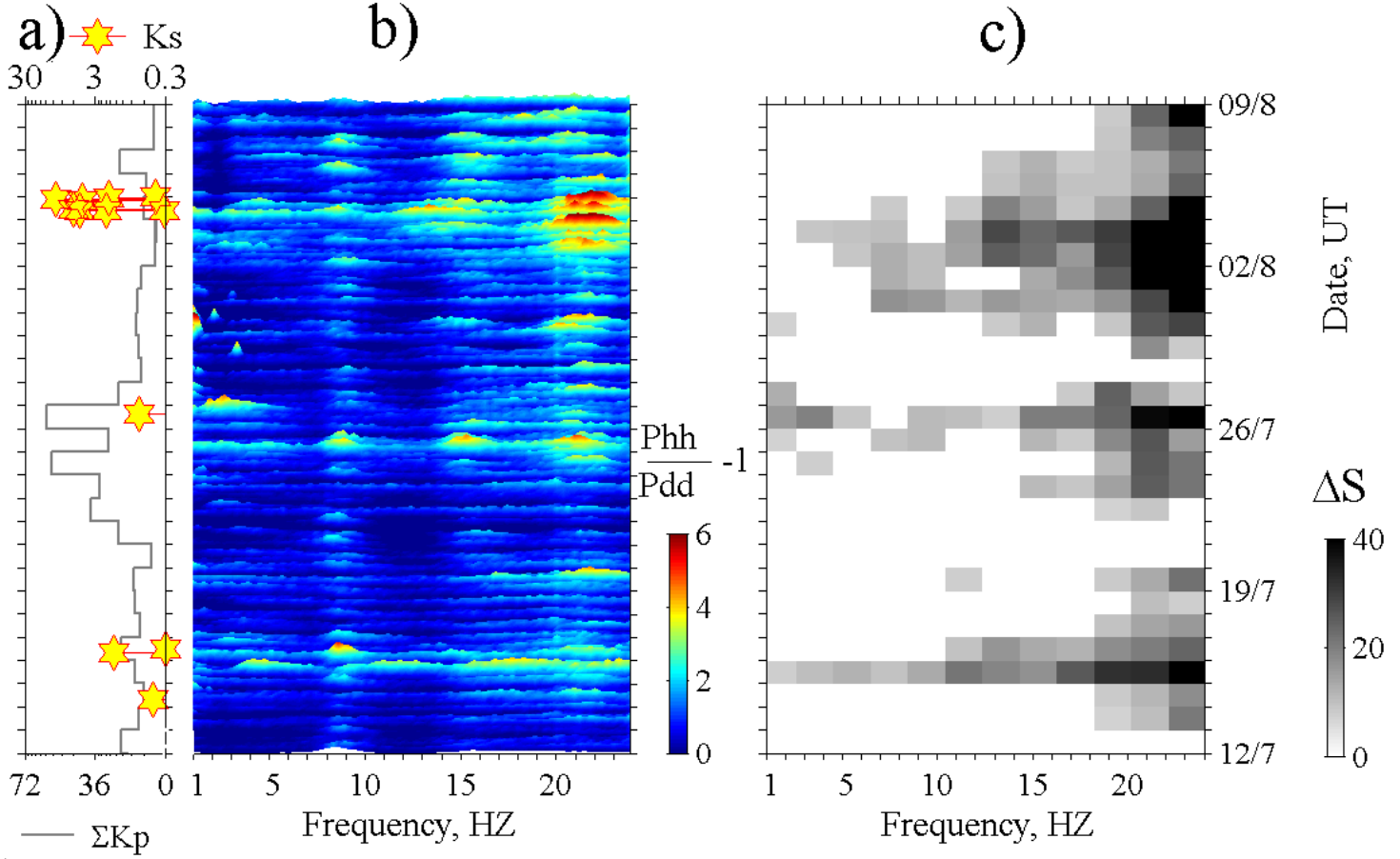

Fig. 3. Seismicity $K_{S}$ and geomagnetic activity $K_{p}$ (a), $P_{h h} / P_{d d}-1$ (b), and $\Delta S$ (c) daily averaged intervals in the frequency band $1-24 \mathrm{~Hz}$ for the interval from 12 July to 8 August 2004

Elements of the polarization matrix are calculated for total field (normal and pulsed parts) using Fourier transform. The choice of the most sensitive parameter is described in details in (Schekotov et al., 2007). Below we use two variants of source azimuth detection with the use of the total field and of its pulsed component.

In the same manner as in (Schekotov et al., 2007) periodgrams are calculated for 256 points (about $5 \mathrm{~s}$ ) and then averaged over 30-min intervals. Mean and rms values used for calculating determinancy $\Delta S$ are calculated using one day sample, including 48 "points", i.e. 30-min spectral parameters.

\subsection{Total field technique}

A success in $\Delta S$ application in (Schekotov et al., 2007) is partly due to a fact that the majority of nearby earthquakes take place eastward from Karymshino. Let now a source be located in the direction turned at some angle $\varphi_{*}$ from $\mathrm{H}$-axis. To find $\varphi_{*}$ let introduce a coordinate system, turned at some angle $\varphi$ from $\mathrm{H}$-axis. Elements of the polarization matrix are then given by

$$
\begin{aligned}
P_{t t} & =P_{h h} \cos ^{2} \varphi+P_{d d} \sin ^{2} \varphi+\operatorname{Re} P_{h d} \sin 2 \varphi \\
P_{r r} & =P_{h h} \sin ^{2} \varphi+P_{d d} \cos ^{2} \varphi-\operatorname{Re} P_{h d} \sin 2 \varphi \\
P_{r t} & =\frac{1}{2}\left(P_{h h}+P_{d d}\right) \sin 2 \varphi+P_{h d} \cos ^{2} \varphi-P_{d h} \sin ^{2} \varphi
\end{aligned}
$$

In the new coordinates the determinancy is given by Eq. (1) where $P_{h h}, P_{d d}, P_{h d}$, and $P_{d h}$ are replaced with $P_{t t}, P_{r r}, P_{t r}$, and $P_{r t}$. Determinancy $\Delta S$, denoted as above, depends on the angle $\varphi$. If the signals are generated mostly by vertical electric currents, the azimuthal component of the magnetic field (perpendicular to the direction to the source) is big in comparison with its radial component (parallel to the direction to the source). Thus $|\Delta S(\varphi)|$ is maximal at $\varphi=\varphi_{*}$, where the angle $\varphi_{*}$ is the angle to a source of radiation.

An example of the distribution averaged over a day of $\Delta S(\varphi)$ in the frequency band $4-6 \mathrm{~Hz}$ is shown in Fig. 1. An arrow indicates the direction to the source.

\subsection{Pulsed component technique}

It was shown in (Schekotov et al., 2007), that there is a difference between preseismic ELF emissions and non-seismic backgraound not only in polarization, but in spectral content as well. Preseismic signal is broadband and enriched with low frequencies. A typical preseismic signal looks like a high-amplitude short pulse. It allows to analyze the pulsed component as follows:

1. Time intervals (of $1 \mathrm{~s}$ duration), for which signal amplitude exceeds some threshold, are selected.

2. For each interval a polarization matrix is calculated using wavelet transform. We take the 4-th order Gauss 


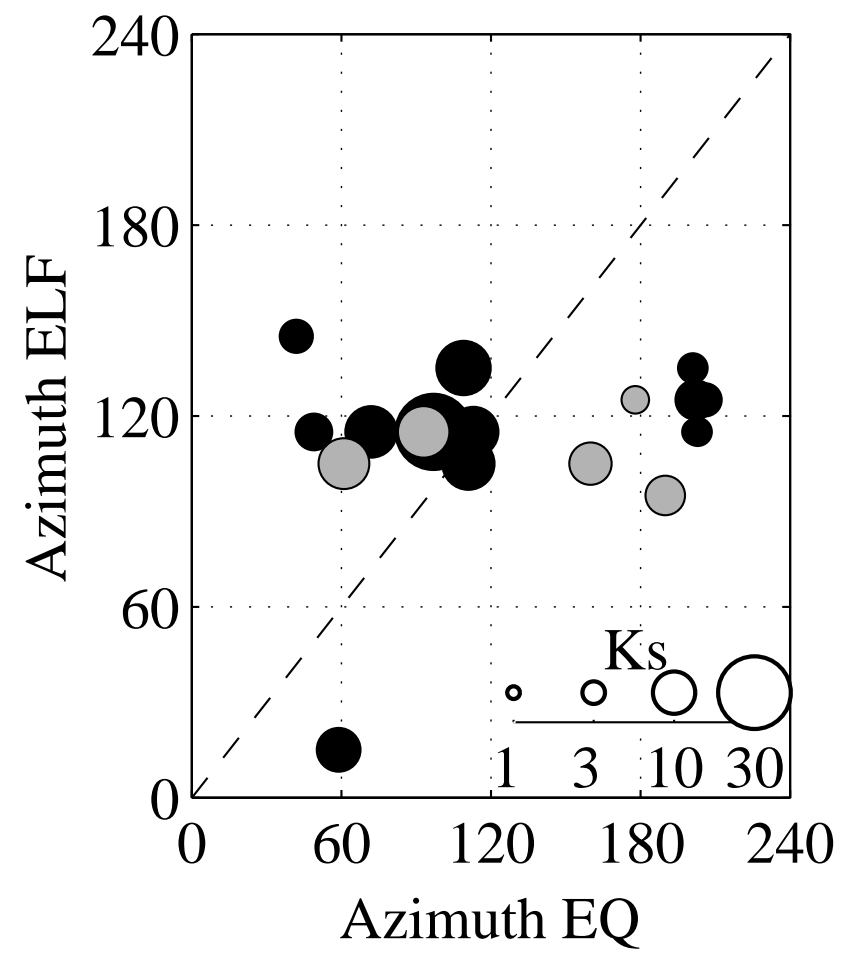

Fig. 4. Source azimuth determined using total-filed technique and EQ azimuths. Black (gray) circles correspond to winter (summer) months

complex wavelet as a basic function to approximate a measured pulse. Such an approximation of asymmetric signal by a symmetric function although results in some additional error, but this inaccuracy is not critical for the purposes of the present study.

3. The parameters of the polarization ellipse, i.e. ellipticity $\beta$ (2) and the angle between the meridian and the ellipse major axis $\theta$, where

$$
\tan (2 \theta)=\frac{2 \operatorname{Re}\left(P_{h d}\right)}{P_{h h}-P_{d d}}
$$

Here $P_{h h}, P_{d d}, P_{h d}$ and $P_{d h}$ are the elements of the wavelet polarization matrix, and the values of $\beta$ (2) and $\theta(4)$ are averaged in the frequency band $4-6 \mathrm{~Hz}$. The probability distribution function (pdf) for the ellipse main axis direction (minor axes corresponds to direction to the source) is calculated for each day. Remain once again, that only selected intervals of over-threshold amplitude are included into this analysis.

An example of data processing is illustrated in Fig. 2. Panels (a) and (b) show records for $H$ and $D$ components, respectively, and panel (c) shows effective total amplitude and amplitude threshold (dashed horizontal line). Panels (d) and (e) show the big axis angle and ellipticity in time domain (gray lines give root mean square deviation). Panels (f) and (g) show the big axis angle and the source azimuth distributions averaged over intervals with amplitudes exceeding the threshold. The figure shows, that during impulses the dispersion of an estimate of axis angle and ellipticity decreases and the polarization of the signal becomes close to linear and ellipses orientations concentrate around some prevailing direction.

The threshold value of amplitude in the selection procedure is determined by the optimization of signal-to-noise ratio. We have taken the threshold of signal amplitude equal to tenfold root square of the dispersion of normal Gaussian field component, calculated as

$\sigma^{2}=\max \left(-\left(\frac{d^{2} \log (\mathrm{pdf})}{d x^{2}}\right)^{-1}\right)$,

where pdf is the probability density function for signal amplitude $x$. Under selected threshold the error in azimuth detection does not exceed 10 degrees.

\section{Results}

An example of EQ time detection with is given in Fig. 3. Seismic activity is shown at panel (a) with the index $K_{S}$, (Schekotov et al., 2007), characterizing seismic power in the observational point. Also at panel (a) we give the global index of geomagnetic activity $K_{p}$ to estimate the level of magnetospheric disturbances. Spectral ratio $P_{h h} / P_{d d}-1$ and determinancy $\Delta S$ in the frequency band 1-24 $\mathrm{HZ}$ are given at panels (b) and (c), respectively. Each of three intervals of high seismic activity is preceded with the interval of $\Delta S$ increased. $\Delta S$ increases significantly $1-5$ days before the main shock, and the timeshift depends on frequency.

At the next step we study the dependence of the direction to the signal source $\theta_{\mathrm{ELF}}$, calculated as described in the previous section, on the direction to the EQ epicenters $\theta_{\mathrm{EQ}}$. The results for total-field technique are shown in Fig. 4. Black (gray) circles correspond to winter (summer) months, respectively. The most probable direction for both is the South-East $\left(100^{\circ}-140^{\circ}\right)$. However, the dispersion is very big and the dependence between signal source and EQ azimuths is by no means meaningful for both seasons.

The picture is quiet different for the pulsed component of the signal (Fig. 5a-d). Each circular panel corresponds to an individual EQ (shown by a circle), and the source azimuth distribution for the pulses, occurred 1-5 days before the EQ are shown by circular histograms. For some events the distribution of $\theta_{\text {ELF }}$ have more than one maximum, but for all of them the main maximum coincides approximately with the direction to the EQ.

However, it is not the only opportunity. A bimodal azimuthal distribution, where the main maximum is nonseismic and the minor maximum is seismic is illustrated in 
$08 / 10 / 2001$

$\mathrm{Ms}=6.2, \mathrm{EpD}=159$

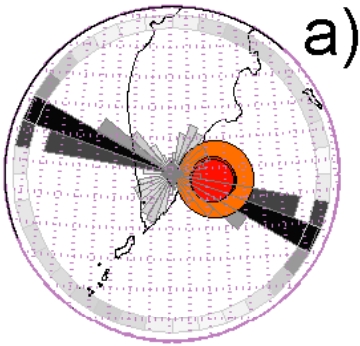

$19 / 03 / 2003$

$\mathrm{Ms}=6.0, \mathrm{EpD}=199$

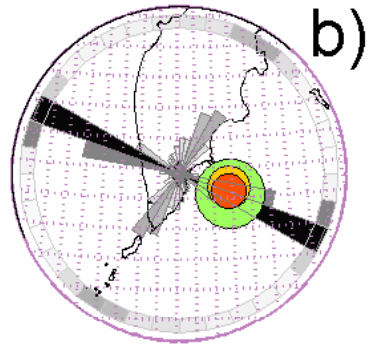

$10 / 08 / 2005$

$\mathrm{Ms}=5.7, \mathrm{EpD}=437$

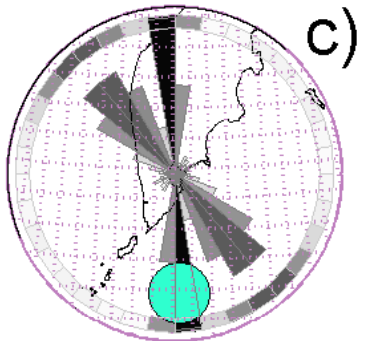

$26 / 11 / 2005$

$\mathrm{Ms}=5.7, \mathrm{EpD}=108$

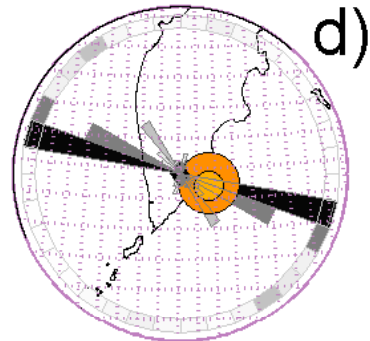

Fig. 5. EQ and signal azimuths, found in pulsed component technique. Each panel corresponds to an individual EQ.

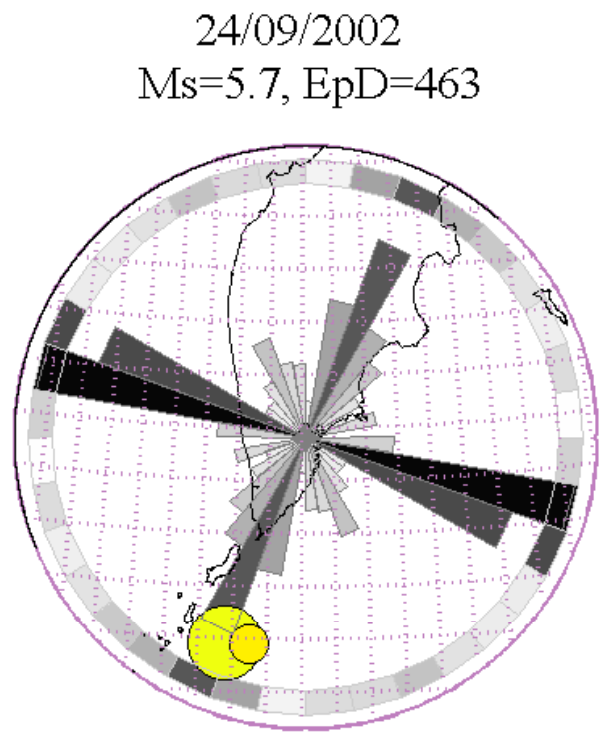

Fig. 6. The same, as in Fig. 5, but for a case of bimodal azimuthal distribution, where the main maximum is non-seismic and the other is seismic.

Fig. 6. The results for all the events analyzed are summarized in Fig. 7, organized identically to Fig. 4. But contrary, to the total field, directions of sources of pulsed component are obviously related to the directions of EQ epicenters. The dispersion of directions is lower for winter months, when local thunderstorm activity is absent.

\section{Discussion and conclusion}

ELF emissions in the frequency band about several hertz, especially its pulsed component, is generated mostly by local sources, namely thunderstorm activity. Periods before earthquakes are characterized by the appearance of additional sources in the EQ preparation zone. The effect is strong enough to be used for the prediction of the time and region of

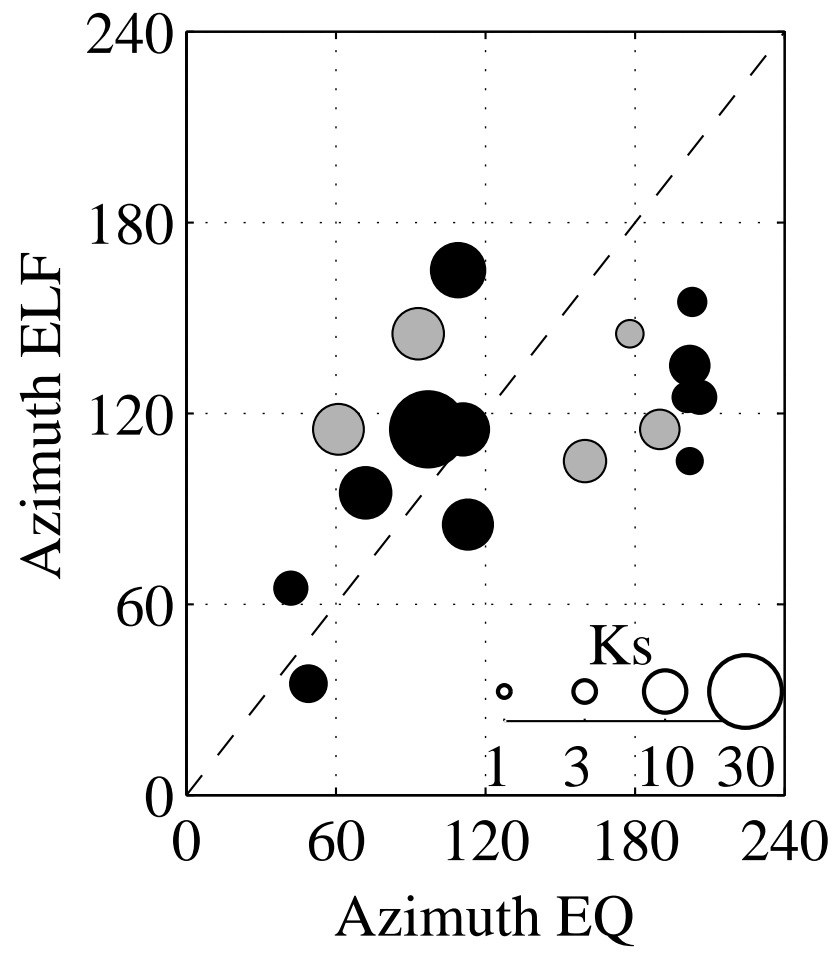

Fig. 7. The same, as Fig. 4, but for the pulsed-component technique.

forthcoming EQ. Local nature of the effect is also confirmed by the fact that the pulsed component is more sensitive to the seismic activity in comparison with total field. The other indirect argument in favor of seismic nature of the observed effect is the better correspondence between the directions to ELF source and EQ epicenters for winter months, when local thunderstorm activity is absent. During local summer emissions, generated by nearby thunderstorms, mask the precursory signal.

The next step to improve the accuracy of EQ detection can be done if multi-point observations become available. A scheme for multi-point observations is given in 


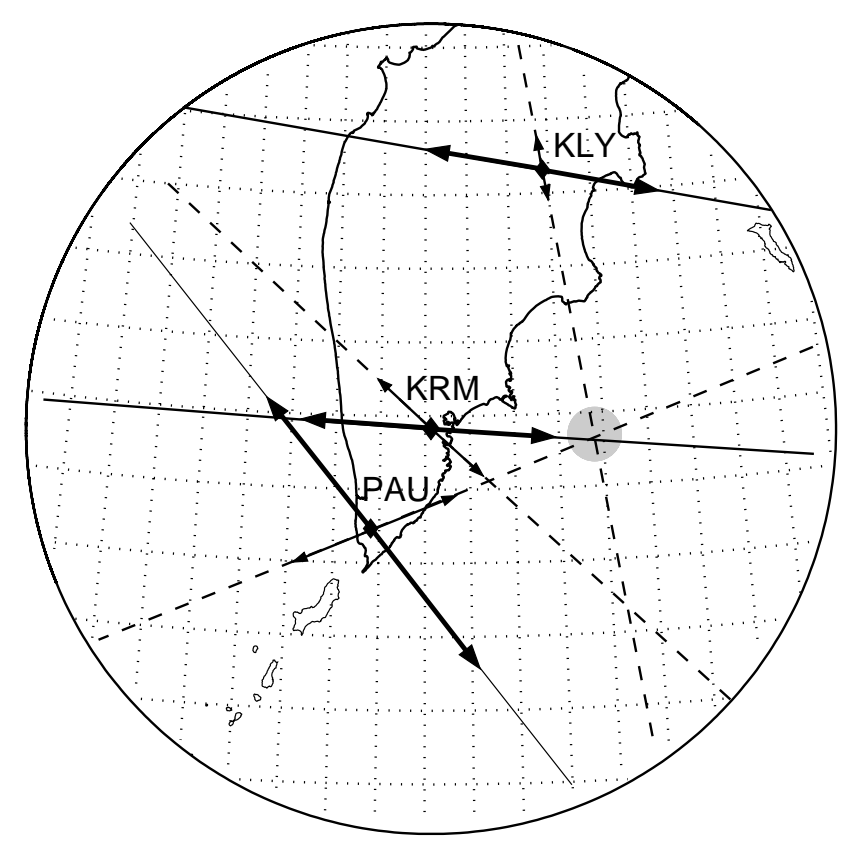

Fig. 8. A scheme of 3-points determination of EQ epicenter position. For each point main mode is shown with solid line, and the second mode is shown with dashed line. The nearest to the observational region point, where three lines cross gives a predicted position of an EQ.

Fig. 8. Along with functioning observatory Karymshino (KRM) two additional points are shown: Pauzhetka (PAU, $51.468^{\circ} \mathrm{N}, 156.815^{\circ} \mathrm{E}$ ) and Klyuchi (KLY, $56.317^{\circ} \mathrm{N}$, $160.858^{\circ} \mathrm{E}$ ). For each point two modes of azimuthal distribution are shown. They are crossed at about 4 degrees eastward from Karymshino, indicating the most seismically active region. This scheme shows that multipoint observations will suppress essentially the error in source detection.

Edited by: P. F. Biagi

Reviewed by: A. Nickolaenko and another anonymous referee

\section{References}

Alperovich, L., Morozov, E., Hayakawa, M., and Hattory, K.: Coherence of the ULF fields in seismoactive zone of Japan, Phys. Chem. Earth, 31, 248-257, 2006.

Fujinawa, Y., Takahashi, K., Matsumoto, T., and Kawakami, N.: Sources of EQ-related VLF electromagnetic signals, in: Atmospheric and ionospheric electromagnetic phenomena associated with earthquakes, edited by: Hayakawa, M., Tokyo, 405-415, 1999.

Kopytenko, Y. A., Ismaguilov, V. S., Hattory, K., and Hayakawa, M.: Determination of earth position of a forthcoming strong EQ using gradients and phase velocities of geomagnetic disturbances, Phys. Chem. Earth, 31, 292-298, 2006.

Molchanov, O. and Hayakawa, M.: Seismo-electromagnetics and related phenomena: History and latest results, TERRAPUB, Tokyo, 190 pp., 2008.

Oike, K. and Yamada, T.: Relationship between shallow earthquakes and electromagnetic noises in LF and VLF ranges, in: Electromagnetic Phenomena Related to Earthquake Prediction, edited by: Hayakawa, M. and Fujinawa, Y., Terra Sci. Pub. Com., Tokyo, 115-130, 1994.

Schekotov, A. Y., Molchanov, O. A., Hayakawa, M., Fedorov, E. N., Chebrov, V. N., Sinitsin, V. I., Gordeev, E. E., Belyaev, G. G., and Yagova, N. V.: ULF/ELF magnetic field variations from atmosphere induced by seismicity, Radio Sci., 42, RS6S90, doi:10.1029/2005RS003441, 2007. 